\title{
Laminar Poroelastic Media Flow
}

\author{
By C. H. Song ${ }^{1}$ and L. H. Huang, ${ }^{2}$ Associate Member, ASCE
}

\begin{abstract}
The continuity and momentum equations of laminar flow through poroelastic media and the sufficient boundary conditions are found in the present paper. The land subsidence equation, Brinkman equation, Darcy's law, and so forth, can be recovered by the simplified governing equations of the present model. A water wave passing over a poroelastic bed is taken as an example of application of this laminar poroelastic media flow model. It is found from the example that, besides the two kinds of longitudinal waves and one kind of transverse wave inside the porous media that the conventional poroelastic model can provide, a second kind of transverse wave is obtained by this model. It is also found that the tangential stress and flow velocity inside the porous bed, according to the laminar poroelastic media flow model, are very different from those obtained by the conventional potential poroelastic media flow model. Finally, the limiting rigid bed solution of the application example, which is useful for the experimental verifications, is also given in this study.
\end{abstract}

\section{INTRODUCTION}

In previous research on water wave interaction with a porous bed, regardless of whether a rigid or deformable skeleton is treated, Darcy's law is applied to describe the friction between the pore fluid and the skeleton of the porous media flow. Although this approach is quite successful in revealing most physical mechanisms by using a potential water wave and Darcy's porous media flow [e.g., see Huang and Song (1993)], due to the use of an irrotational wave and Darcy's law, slip at the interface of water and porous bed cannot be avoided. This causes handicaps, especially when correct shear stress at the interface is important (e.g., in the determination of ripple formation at the alluvial channel bed).

Some works try to remedy the unreasonable slip phenomenon at the porous interface by introducing fluid viscosity into the water wave [e.g., Hunt (1959); Murray (1965)]; however, because Darcy's law is only a first-order differentiation in space, an overdetermined boundary condition occurs at the interface. Liu (1973) adopted the boundary layer approach and velocity correction due to viscosity in order to satisfy velocity continuity at the interface, but continuous tangential stress is still not satisfied.

Two successful previous methods can be found to rectify the unreasonable slip phenomenon at the porous boundary. The first is proposed by Beavers and Joseph (1967). In their study, parallel viscous flow and Darcy's porous media flow are employed to portray the homogeneous upper flow and lower porous media flow, respectively, while the following ingenious partial-slip boundary condition is suggested to overcome the overdetermined boundary condition:

$$
\left.\frac{\partial u}{\partial y}\right|_{y=0^{+}}=\frac{\alpha}{\sqrt{k_{p}}}\left(u-u_{D}\right)
$$

In (1), $u$ is the velocity of the upper layer flow; $u_{D}$ is $u$ at boundary $y=0^{+} ; k_{p}$ is the specific permeability of the lower porous layer; and $\alpha$ is a parameter to be determined empirically.

The second successful method is treating homogeneous fluid as viscous flow, while handling the porous media flow by the so-called Brinkman equation (Brinkman 1947)

\footnotetext{
${ }^{1}$ Assoc. Res., Tainan Hydr. Lab., Nat. Cheng Kung Univ., Tainan, Taiwan, ROC.

${ }^{2}$ Prof., Dept. of Civ. Engrg. and Hydrotech Res. Inst., Nat. Taiwan Univ., Taipei, Taiwan, ROC.

Note. Associate Editor: Alexander Cheng. Discussion open until September 1, 2000. To extend the closing date one month, a written request must be filed with the ASCE Manager of Journals. The manuscript for this paper was submitted for review and possible publication on January 26, 1999. This paper is part of the Journal of Engineering Mechanics, Vol. 126, No. 4, April, 2000. CASCE, ISSN 0733-9399/00/0004-03580366/\$8.00 + \$.50 per page. Paper No. 20140.
}

$$
-\nabla P+\mu \nabla^{2} \mathbf{u}-\frac{\mu}{k_{p}} \mathbf{u}=\mathbf{0}
$$

where $P, \mathbf{u}$ are the pressure (with static pressure included) and velocity vector of flow in the porous media, respectively, and $\mu$ is the fluid viscosity. Because (2) is a second-order differential equation, the Brinkman equation with proper boundary conditions [which are not given in Brinkman (1947)] indeed can handle the overdetermined boundary problem at the fluid/ porous-media interface.

Besides the defect of the empirically determined parameter of Beavers and Joseph's method and the unclear boundary conditions of Brinkman's proposal, the use of these two methods is still rather restricted. For example, when the inertial effect of the porous media flow is important (e.g., wave propagation in the porous media), or when the porous medium is deformable (i.e., the porous skeleton is no longer rigid), the above-mentioned two ways, again, are both inadequate.

The present work will propose a model of viscous porous media flow with an elastically deforming porous skeleton. We will present the governing equations and their proper boundary conditions and then apply this model to investigate the problem of a viscous water wave passing over a poroelastic bed.

\section{GOVERNING EQUATIONS AND BOUNDARY CONDITIONS}

\section{Continuity Equations}

In saturated porous media flow, if $\rho_{s}, \rho_{f}$ are the solid and fluid densities, respectively; $\dot{\mathbf{d}}, \mathbf{U}$ are the solid and fluid velocity vectors; and $n$ is porosity, then the continuity equations of the solid and fluid, respectively, are [Verruijt (1969), etc.]

$$
\begin{gathered}
\frac{\partial}{\partial t}\left[(1-n) \rho_{s}\right]+\nabla \cdot\left[(1-n) \rho_{s} \mathbf{d}\right]=0 \\
\frac{\partial}{\partial t}\left(n \rho_{f}\right)+\nabla \cdot\left(n \rho_{f} \mathbf{U}\right)=0
\end{gathered}
$$

Combining (3) and (4), we get

$$
\begin{gathered}
\frac{(1-n)}{\rho_{s}}\left(\frac{\partial \rho_{s}}{\partial t}+\dot{\mathbf{d}} \cdot \nabla \rho_{s}\right)+\frac{n}{\rho_{f}}\left(\frac{\partial \rho_{f}}{\partial t}+\mathbf{U} \cdot \nabla \rho_{f}\right) \\
=-[(1-n) \nabla \cdot \dot{\mathbf{d}}+n \nabla \cdot \mathbf{U}]-(\mathbf{U}-\dot{\mathbf{d}}) \cdot \nabla n
\end{gathered}
$$

Note that we ignore the $\partial n / \partial t$ term at present for convenience because most of the time it is not important [e.g., Mei and Foda (1981)].

Grain compressibility usually is neglected-that is, $d \rho_{s} / d t$ $=0\left[\right.$ Mei and Foda (1981)], and we may assume $\nabla \rho_{f}=\nabla n=$ 0; further, following Verruijt's (1969) suggestion, we get 


$$
\frac{\partial \rho_{f}}{\partial t}=\frac{\rho_{f}}{K} \frac{\partial P^{\prime}}{\partial t}
$$

where $K$ is the static bulk modulus of compressibility of fluid in the porous media, and $P^{\prime}$ is the perturbed fluid pressure caused by fluid dilatation. Therefore, (5) becomes

$$
\frac{\partial P^{\prime}}{\partial t}=-\frac{K}{n}[(1-n) \nabla \cdot \dot{\mathbf{d}}+n \nabla \cdot \mathbf{U}]
$$

which is the so-called storage equation.

\section{Momentum Equations}

From the first law of thermodynamics, we may have

$$
\frac{\delta Q}{d t}+\frac{\delta W}{d t}=\frac{\delta E}{d t}
$$

inside the porous media. The terms $Q$ and $W$ are heat and work added to the system, while $E$ is the internal energy of the system. For the adiabatic process

$$
\frac{\delta Q}{d t}=0
$$

The time rate of change of work

$$
\begin{aligned}
\frac{\partial W}{d t} & =\iint_{S}\left(\boldsymbol{\sigma}_{s} \cdot \dot{\mathbf{d}}+\boldsymbol{\sigma}_{f} \cdot \mathbf{U}\right) \cdot \mathbf{n} d S \\
& +\iiint_{V}\left[(1-n) \rho_{s} \mathbf{g} \cdot \dot{\mathbf{d}}+n \rho_{f} \mathbf{g} \cdot \mathbf{U}\right] d V
\end{aligned}
$$

can be written as

$$
\begin{aligned}
& \frac{\delta W}{d t}=\iiint_{V}\left[\nabla \cdot \boldsymbol{\sigma}_{s} \cdot \dot{\mathbf{d}}+\nabla \cdot \boldsymbol{\sigma}_{f} \cdot \mathbf{U}+\boldsymbol{\sigma}_{s}: \nabla \dot{\mathbf{d}}+\boldsymbol{\sigma}_{f}: \nabla \mathbf{U}\right. \\
& \left.\quad+(1-n) \rho_{s} \mathbf{g} \cdot \dot{\mathbf{d}}+n \rho_{f} \mathbf{g} \cdot \mathbf{U}\right] d V
\end{aligned}
$$

after applying the divergence theorem to the work done by stress on the surface. In (11), $\boldsymbol{\sigma}_{s}$ and $\boldsymbol{\sigma}_{f}$ are the stress tensors of the solid skeleton and fluid; $\mathbf{g}$ is gravitational acceleration; and the colon represents the scalor product of two secondorder tensors.

The time rate of change of internal energy is

$$
\begin{aligned}
\frac{\delta E}{d t} & =\iiint_{V}\left\{\frac{1}{2} \frac{d}{d t}\left[(1-n) \boldsymbol{\rho}_{s} \dot{\mathbf{d}} \cdot \dot{\mathbf{d}}+n \rho_{f} \mathbf{U} \cdot \mathbf{U}\right]\right. \\
& \left.+\left[\boldsymbol{\sigma}_{s}: \nabla \dot{\mathbf{d}}+\boldsymbol{\sigma}_{f}: \nabla \mathbf{U}\right]+F(\kappa) \frac{\mu n^{2}}{k_{p}}(\dot{\mathbf{d}}-\mathbf{U}) \cdot(\dot{\mathbf{d}}-\mathbf{U})\right\} d V
\end{aligned}
$$

where $F(\kappa)$ is the frequency correction factor defined in Biot (1956); $\mu$ is the dynamic viscosity of the fluid; and $k_{p}$ is the specific permeability of the porous medium. Note that because there is no relative slipping between solid skeleton and fluid, Kirchoff's coupling kinetic energy (i.e., added mass effect) vanishes in the first term of the righthand side of (12), and the symmetry of the stress tensors is applied to the strain energy term [i.e., the second term at the righthand side of (12)], while the remaining mechanical energy due to surface contact is still the same as in Biot (1962). Applying continuity equations (3) and (4) to (12), we get

$$
\begin{aligned}
\frac{\delta E}{d t} & =\iiint_{V}\left\{(1-n) \rho_{s} \dot{\mathbf{d}} \cdot \ddot{\mathbf{d}}+n \rho_{f} \mathbf{U} \cdot \dot{\mathbf{U}}+\sigma_{s}: \nabla \dot{\mathbf{d}}+\sigma_{f}: \nabla \mathbf{U}\right. \\
& \left.+F(\kappa) \frac{\mu n^{2}}{k_{p}}(\dot{\mathbf{d}}-\mathbf{U}) \cdot(\dot{\mathbf{d}}-\mathbf{U})\right\} d V
\end{aligned}
$$

Substituting (9), (11), and (13) in (8), we finally get

$$
\begin{aligned}
& \iiint_{V}\left\{\left[\nabla \cdot \boldsymbol{\sigma}_{s}+(1-n) \rho_{s} \mathbf{g}-(1-n) \rho_{s} \ddot{\mathbf{d}}\right.\right. \\
& \left.+F(\kappa) \frac{\mu n^{2}}{k_{p}}(\mathbf{U}-\dot{\mathbf{d}})\right] \cdot \dot{\mathbf{d}}+\left[\nabla \cdot \boldsymbol{\sigma}_{f}+n \rho_{f} \mathbf{g}-n \rho_{f} \mathbf{U}\right. \\
& \left.\left.\quad-F(\kappa) \frac{\mu n^{2}}{k_{p}}(\mathbf{U}-\dot{\mathbf{d}})\right] \cdot \mathbf{U}\right\} d V=0
\end{aligned}
$$

Therefore, (14) indicates the momentum equations as

$$
\begin{gathered}
\nabla \cdot \boldsymbol{\sigma}_{s}+(1-n) \rho_{s} \mathbf{g}=(1-n) \rho_{s} \ddot{\mathbf{d}}-F(\kappa) \frac{\mu n^{2}}{k_{p}}(\mathbf{U}-\dot{\mathbf{d}}) \\
\nabla \cdot \boldsymbol{\sigma}_{f}+n \rho_{f} \mathbf{g}=n \rho_{f} \dot{\mathbf{U}}+F(\kappa) \frac{\mu n^{2}}{k_{p}}(\mathbf{U}-\dot{\mathbf{d}})
\end{gathered}
$$

Considering the mass coupling effect for the potential flow and getting rid of the viscous effect of the fluid, we find that (15) and (16) recover the conventional theory of poroelasticity (Biot 1962). On the other hand, by neglecting the inertial and viscous effects of the fluid, the equilibrium equations of land subsidence are obtained; assuming a rigid solid skeleton, neglecting inertial effect, and letting $F(\kappa)=1$, (16) may give the Brinkman equation [i.e., (2)]; further, neglecting fluid viscosity, we get the following Darcy's law:

$$
-\nabla P+\rho_{f} g=\frac{\mu n}{k_{p}} \mathbf{U}
$$

Also notice that (15) and (16) are similar to the equations derived in Katsube and Carroll (1987), based on mixture theory. However, in Katsube and Carroll (1987), there is an unrealistic torsion term for the fluid (fluid obviously does not contain "torque").

If there is a steady streaming flow through the porous media, we may let

$$
\begin{aligned}
\mathbf{d}(\mathbf{x}, t) & =\mathbf{d}^{0}(\mathbf{x})+\mathbf{d}^{\prime}(\mathbf{x}, t) \\
\mathbf{U}(\mathbf{x}, t) & =\mathbf{U}^{0}(\mathbf{x})+\mathbf{U}^{\prime}(\mathbf{x}, t)
\end{aligned}
$$

with $\left|\mathbf{U}^{\prime}\right| \ll\left|\mathbf{U}^{0}\right|,\left|\mathbf{d}^{\prime}\right| \ll<\left|\mathbf{d}^{0}\right|$. Then, from (15) and (16), the equations for the stationary deformed solid skeleton and steady streaming viscous flow are

$$
\begin{gathered}
\nabla \cdot \boldsymbol{\sigma}_{s}^{0}+(1-n) \rho_{s} \mathbf{g}=-F(\kappa) \frac{\mu n^{2}}{k_{p}} \mathbf{U}^{0} \\
\nabla \cdot \boldsymbol{\sigma}_{f}^{0}+n \rho_{f} \mathbf{g}=n \rho_{f}\left(\mathbf{U}^{0} \cdot \nabla\right) \mathbf{U}^{0}+F(\kappa) \frac{\mu n^{2}}{k_{p}} \mathbf{U}^{0}
\end{gathered}
$$

On the other hand, the linear equations of motion of the solid and fluid for the remaining disturbance are

$$
\begin{gathered}
\nabla \cdot \boldsymbol{\sigma}_{s}^{\prime}=(1-n) \rho_{s} \frac{\partial^{2} \mathbf{d}^{\prime}}{\partial t^{2}}-F(\kappa) \frac{\mu n^{2}}{k_{p}}\left(\mathbf{U}^{\prime}-\frac{\partial \mathbf{d}^{\prime}}{\partial t}\right) \\
\nabla \cdot \sigma_{f}^{\prime}=n \rho_{f} \frac{\partial^{2} \mathbf{U}^{\prime}}{\partial t^{2}}+F(\kappa) \frac{\mu n^{2}}{k_{p}}\left(\mathbf{U}^{\prime}-\frac{\partial \mathbf{d}^{\prime}}{\partial t}\right)
\end{gathered}
$$

In (22) and (23), if the generalized Hooke's law for the solid skeleton and Newton's law for fluids are valid, stress tensors can be written as

$$
\begin{gathered}
\boldsymbol{\sigma}_{s}^{\prime}=\boldsymbol{\tau}_{s}^{\prime}-(1-n) P^{\prime} \mathbf{I} \\
\boldsymbol{\sigma}_{f}^{\prime}=n \boldsymbol{\tau}_{f}^{\prime}-n P^{\prime} \mathbf{I}
\end{gathered}
$$

where I is the identity matrix, while the effective stress for the solid skeleton and the shear stress of the fluid are 


$$
\begin{gathered}
\boldsymbol{\tau}_{s}^{\prime}=G\left[\nabla \mathbf{d}^{\prime}+\left(\nabla \mathbf{d}^{\prime}\right)^{T}\right]+\lambda\left(\nabla \cdot \mathbf{d}^{\prime}\right) \mathbf{I} \\
\boldsymbol{\tau}_{f}^{\prime}=\mu\left[\nabla \mathbf{U}^{\prime}+\left(\nabla \mathbf{U}^{\prime}\right)^{T}\right]+\mu^{\prime}\left(\nabla \cdot \mathbf{U}^{\prime}\right) \mathbf{I}
\end{gathered}
$$

with $G, \lambda$ as Lambe's constants of elasticity, and $\mu, \mu^{\prime}$ as first and second fluid viscosity; superscript $T$ denotes the transpose of matrix.

Eqs. (7), (22), and (23), together with the constitutive laws (24)-(27), will be the governing equations when later we deal with the application example of a viscous water wave passing over a poroelastic bed.

\section{Boundary Conditions}

Referring to Deresiewicz and Skalak (1962), the sufficient conditions (except kinematic boundary conditions) can be derived. Let the adjacent boundary between two porous media be $S_{c}$ and the unit normal vectors be $\boldsymbol{v}^{(1)}$ and $\boldsymbol{v}^{(2)}\left(\boldsymbol{v}^{(1)}=-\boldsymbol{v}^{(2)}\right)$; then the continuity of the time rate of change of work on the surface is [superscript $(k)$ indicates the summation in $k$ ]

$$
\iint_{S c}\left[\boldsymbol{\sigma}_{s}^{(k)} \cdot \dot{\mathbf{d}}^{(k)}+\boldsymbol{\sigma}_{f}^{(k)} \cdot \mathbf{U}^{(k)}\right] \cdot \boldsymbol{v}^{(k)} d A=0 ; \quad k=1,2
$$

On the other hand, the continuity of the fluid flux through $S_{c}$ is

$$
\iint_{S c} n^{(k)}\left(\mathbf{U}^{(k)}-\dot{\mathbf{d}}^{(k)}\right) \cdot \boldsymbol{v}^{(k)} d A=0 ; \quad k=1,2
$$

Combining (28) and (29), we get

$$
\begin{aligned}
& \iint_{S c}\left[\left(\boldsymbol{\sigma}_{s}^{(k)}+\boldsymbol{\sigma}_{f}^{(k)}\right) \cdot \dot{\mathbf{d}}^{(k)}+\frac{\boldsymbol{\sigma}_{f}^{(k)}}{n^{(k)}} \cdot\left(\mathbf{U}^{(k)}-\dot{\mathbf{d}}^{(k)}\right)+n^{(k)}\left(\mathbf{U}^{(k)}-\dot{\mathbf{d}}^{(k)}\right)\right] \\
& \cdot \boldsymbol{v}^{(k)} d A=0 ; \quad k=1,2
\end{aligned}
$$

which indicates that (except for the kinematic boundary condition) there are eight physical variables that should be continuous at the porous interface: $\boldsymbol{v} \cdot\left(\boldsymbol{\sigma}_{s}+\boldsymbol{\sigma}_{f}\right) \cdot \boldsymbol{v} ; t \cdot\left(\boldsymbol{\sigma}_{s}+\boldsymbol{\sigma}_{f}\right) \cdot$ $\boldsymbol{v} ; \boldsymbol{v} \cdot \boldsymbol{\sigma}_{f} / n \cdot \boldsymbol{v} ; t \cdot \boldsymbol{\sigma}_{f} / \mathbf{n} \cdot \boldsymbol{v} ; \dot{\mathbf{d}} \cdot \boldsymbol{v} ; \dot{\mathbf{d}} \cdot t ; \boldsymbol{v} \cdot n(\mathbf{U}-\dot{\mathbf{d}}) ;$ and $t \cdot n(\mathbf{U}-$ $\dot{\mathbf{d}})$, where $t$ is the unit tangent vector. The physical meanings, in sequence, are the continuities of (1) normal total stress; (2) tangential total stress; (3) normal fluid stress; (4) tangential fluid stress; (5) normal solid velocity; (6) tangential solid velocity; (7) normal fluid flux; and (8) tangential relative flow velocity.

For example, if medium 1 is a homogeneous fluid while medium 2 is a porous medium, then, besides the kinematic boundary condition (which will be used to determine the shape of the interface if it is deformable), we'll need the continuities of items (1)-(4), (7), and (8); if medium 1 is a homogeneous fluid, while medium 2 is a rigid porous medium, then the continuities of items (3), (4), (7), and (8) are required.

\section{APPLICATION: VISCOUS WATER WAVE PASSING OVER POROELASTIC BED}

Huang, Chiang, and Song (1996) applied the above theory and reinvestigated Beavers and Joseph's (1967) problem of laminar channel flow passing over a rigid porous bed. The result of Huang, Chiang, and Song (1996) agrees with the experimental data of Beavers and Joseph (1967) perfectly, and a conventional empirical parameter due to partial slip at the interface [i.e., $\alpha$ in (1)] is derived explicitly, based on the present theory. Recently, in Liu, Davis, and Downing (1996), viscous water waves over a rigid permeable bed were studied both theoretically and experimentally. However, since the orders of magnitude of physical parameters are so different between "hard" and "soft" porous media [e.g., Chen, Huang, and Song (1997)], general solutions that are not restricted to

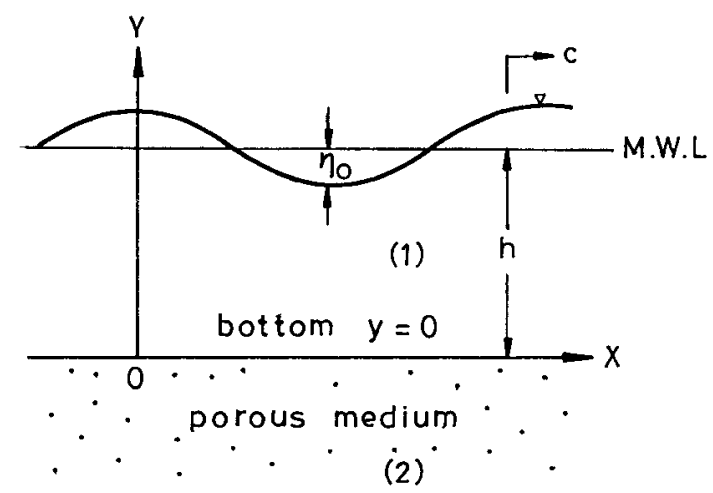

FIG. 1. Schematic Diagram of Water Wave Passing over Poroelastic Bed

a rigid porous medium are worth studying. Therefore, without assuming a rigid bed, a viscous water wave passing over a poroelastic bed will be explored by using the present theory in the following.

The schematic diagram of the problem is indicated in Fig. 1 , in which a linear water wave propagating along a constant depth channel and passing over a porous bed of infinite thickness is studied. Fluid viscosities of water in both the channel and porous bed are considered, and the solid skeleton of the porous bed is assumed to deform elastically. From $y=0$ to $y$ $=h$, indicated as in region (1), there is undisturbed channel water, while from $y=0$ to $y \rightarrow-\infty$, denoted as in region (2), a porous bed is assigned.

\section{Governing Equations of Channel Flow}

Getting rid of oscillatory time factor $e^{-i \omega t}$, with $\omega$ as the angular frequency, the time-independent perturbed velocity of the water in the channel can be divided into an irrotational and a rotational part, as follows:

$$
\mathbf{V}^{(1)}=\nabla \Phi_{1}^{(1)}+\nabla \times \mathbf{H}_{1}^{(1)}
$$

Thus, the continuity equation becomes

$$
\nabla^{2} \Phi_{1}^{(1)}=0
$$

shear stress becomes $\left(\boldsymbol{\sigma}_{f}^{(1)}=\boldsymbol{\tau}_{f}^{(1)}-P \mathbf{I}\right)$

$$
\boldsymbol{\tau}_{f}^{(1)}=\mu\left[2 \nabla \nabla \Phi_{1}^{(1)}+\nabla\left(\nabla \times \mathbf{H}_{1}^{(1)}\right)+\left\{\nabla\left(\nabla \times \mathbf{H}_{1}^{(1)}\right\}^{T}\right]\right.
$$

and the linear momentum equation (after getting rid of hydrostatic pressure) becomes

$$
\begin{gathered}
i \rho_{f} \omega \Phi_{1}^{(1)}+P^{(1)}=0 \\
-i \rho_{f} \omega\left(\nabla \times \mathbf{H}_{1}^{(1)}\right)=\mu \nabla^{2}\left(\nabla \times \mathbf{H}_{1}^{(1)}\right)
\end{gathered}
$$

In (33) and (35), we only consider the first fluid viscosity $\mu$, and the second fluid viscosity $\mu^{\prime}$ will be neglected hereafter.

Since we are in 2D Cartesian coordinates (i.e., $\mathrm{x}-\mathrm{y}$ plane), we may let

$$
\mathbf{H}_{1}^{(1)}=\Phi_{2}^{(1)} e_{z}
$$

with $e_{z}$ as the unit vector, which is perpendicular to the $\mathrm{x}-\mathrm{y}$ plane. Hence, (35) can be written as

$$
\begin{gathered}
\nabla^{2} \Phi_{2}^{(1)}+k_{w}^{2} \Phi_{2}^{(1)}=0 \\
k_{w}^{2}=\frac{i \rho_{f} \omega}{\mu}
\end{gathered}
$$

\section{Governing Equations of Poroelastic Bed}

The governing equations of the poroelastic bed can be obtained by referring to the aforementioned sections. Following 
the same procedures as those listed in Appendix A of Huang and Chwang (1990), the equivalent time-independent equations (with the same notations as time-dependent variables and prime being got rid of for simplicity) with the superscript (2) added to denote the variables in region (2) become as follows:

$$
\begin{gathered}
\nabla^{2} \Phi_{j}^{(2)}+k_{j}^{2} \Phi_{j}^{(2)}=0 ; \quad j=1,2 \\
k_{1}^{2}+k_{2}^{2}=\frac{A_{1}\left(2 E+R_{0}\right)-2 A_{2} Q+(2 G+A) A_{3}}{(2 G+A)\left(2 E+R_{0}\right)-Q^{2}} \\
k_{1}^{2} k_{2}^{2}=\frac{A_{1} A_{3}-A_{2}^{2}}{(2 G+A)\left(2 E+R_{0}\right)-Q^{2}} \\
\nabla^{2} \mathbf{H}_{j}^{(2)}+k_{j}^{2} \mathbf{H}_{j}^{(2)}=\mathbf{0} ; j=3,4 \\
k_{3}^{2}+k_{4}^{2}=\frac{E A_{1}+G A_{3}}{E G} \\
k_{3}^{2} k_{4}^{2}=\frac{-A_{2}^{2}+A_{1} A_{3}}{E G}
\end{gathered}
$$

where

$$
\begin{gathered}
\mathbf{d}=\nabla \Phi_{1}^{(2)}+\nabla \Phi_{2}^{(2)}+\nabla \times \mathbf{H}_{3}^{(2)}+\nabla \times \mathbf{H}_{4}^{(2)} \\
\mathbf{D}=\alpha_{1} \nabla \Phi_{1}^{(2)}+\alpha_{2} \nabla \Phi_{2}^{(2)}+\alpha_{3} \nabla \times \mathbf{H}_{3}^{(2)}+\alpha_{4} \nabla \times \mathbf{H}_{4}^{(2)}
\end{gathered}
$$

$\mathbf{d}$ and $\mathbf{D}$ are displacement vectors of solid skeleton and fluid; and

$$
\begin{gathered}
\alpha_{j}=\frac{-k_{j}^{2}\left[(2 G+A)\left(2 E+R_{0}\right)-Q^{2}\right]+\left(A_{1}\left(2 E+R_{0}\right)-A_{2} Q\right]}{Q A_{3}-\left(2 E+R_{0}\right) A_{2}} ; \\
j=1,2 \\
\alpha_{j}=\frac{-k_{j}^{2} G-A_{1}}{A_{2}} ; j=3,4
\end{gathered}
$$

where

$$
\begin{gathered}
A=\lambda+Q q \\
Q=K(1-n) \\
q=(1-n) / n \\
E=-i \omega \mu n \\
R_{0}=n K \\
A_{1}=\omega\left[\begin{array}{c}
(1-n) \rho_{s} \omega+i F(\kappa) \frac{\mu n^{2}}{k_{p}}
\end{array}\right] \\
A_{2}=-i \omega F(\kappa) \frac{\mu n^{2}}{k_{p}} \\
A_{3}=\omega\left(n \rho_{f} \omega+i F(\kappa) \frac{\mu n^{2}}{k_{p}}\right)
\end{gathered}
$$

and

$$
\begin{aligned}
& P^{(2)}=-\frac{\mathbf{K}}{n}\left[\left(1-n+\alpha_{1} n\right) k_{1}^{2} \Phi_{1}^{(2)}+\left(1-n+\alpha_{2} n\right) k_{2}^{2} \Phi_{2}^{(2)}\right] \\
& \tau_{s}^{(2)}=G\left[2 \nabla \nabla \Phi_{1}^{(2)}+2 \nabla \nabla \Phi_{2}^{(2)}+\nabla\left(\nabla \times \mathbf{H}_{3}^{(2)}\right)+\left\{\nabla\left(\nabla \times \mathbf{H}_{3}^{(2)}\right\}^{T}\right.\right. \\
& \quad+\nabla\left(\nabla \times \mathbf{H}_{4}^{(2)}\right)+\left\{\nabla\left(\nabla \times \mathbf{H}_{4}^{(2)}\right\}^{T}\right] \\
& \quad-\lambda\left(k_{1}^{2} \Phi_{1}^{(2)}+k_{(2)}^{2} \Phi_{2}^{(2)}\right) \mathbf{I} \\
& \tau_{f}^{(2)}=\mu\left[2 \alpha_{1} \nabla \nabla \dot{\Phi}_{1}^{(2)}+2 \alpha_{2} \nabla \nabla \dot{\Phi}_{2}^{(2)}+\alpha_{3}\left(\nabla\left(\nabla \times \dot{\mathbf{H}}_{3}^{(2)}\right)\right.\right. \\
& +\left\{\nabla\left(\nabla \times \dot{\mathbf{H}}_{3}^{(2)}\right\}^{T}\right)+\alpha_{4}\left(\nabla\left(\nabla \times \dot{\mathbf{H}}_{4}^{(2)}\right)+\left\{\nabla\left(\nabla \times \dot{\mathbf{H}}_{4}^{(2)}\right\}^{T}\right)\right] \\
& \quad\left(\mu^{\prime} \text { is neglected }\right)
\end{aligned}
$$

$\boldsymbol{\sigma}_{s}$ and $\boldsymbol{\sigma}_{f}$ are given by (24) and (25), respectively. Again, for a $2 \mathrm{D}$ problem in Cartesian coordinates $\left(\mathrm{x}-\mathrm{y}\right.$ plane), $\mathbf{H}_{j}^{(2)}$ can be written as $\Phi_{j}^{(2)} e_{z}$.

Note that, due to the inclusion of fluid viscosity, we get two longitudinal waves and two transverse waves instead of two longitudinal waves and one transverse wave according to the conventional Biot's theory of elasticity (Huang and Song 1993).

\section{Boundary Conditions}

The conventional kinematic boundary condition on the free surface $(y=h)$ is

$$
\Phi_{1, y}^{(1)}-\Phi_{2, x}^{(1)}=-i \omega \eta
$$

where $\eta$ is the time-independent displacement at the free surface. The dynamic boundary condition on the free surface $(y$ $=h$ ), continuities of normal and tangential stresses, can be written as

$$
\begin{gathered}
-i \rho_{f} \omega \Phi_{1}^{(1)}+2 \mu\left(\Phi_{1, y y}^{(1)}-\Phi_{2, x y}^{(1)}\right)=-\rho_{f} g \eta \\
2 \Phi_{1, x y}^{(1)}+\Phi_{2, y y}^{(1)}-\Phi_{2, x x}^{(1)}=0
\end{gathered}
$$

As for the boundary conditions at the interface of the porous bed $(y=0)$, we find that, except for the kinematic boundary condition, which is only used for finding small sand waves, we may have continuities of (1) normal fluid flux; (2) tangential relative fluid velocity; (3) normal fluid stress; (4) tangential fluid stress; (5) normal total stress; and (6) tangential total stress. They are listed, in sequence, as follows:

$$
\begin{aligned}
& \Phi_{1, y}^{(1)}-\Phi_{2, x}^{(1)}=-i \omega\left[\left(1-n+\alpha_{1} n\right) \Phi_{1, y}^{(2)}+\left(1-n+\alpha_{2} n\right) \Phi_{2, y}^{(2)}\right. \\
& \left.\quad-\left(1-n+\alpha_{3} n\right) \Phi_{3, x}^{(2)}-\left(1-n_{0}+\alpha_{4} n_{0}\right) \Phi_{4, x}^{(2)}\right] \\
& \Phi_{1, x}^{(1)}-\Phi_{2, y}^{(1)}=-i \omega\left[\left(1-n+\alpha_{1} n\right) \Phi_{1, x}^{(2)}+\left(1-n+\alpha_{2} n\right) \Phi_{2, x}^{(2)}\right. \\
& \left.\quad+\left(1-n+\alpha_{3} n\right) \Phi_{3, y}^{(2)}+\left(1-n_{0}+\alpha_{4} n\right) \Phi_{4, y}^{(2)}\right] \\
& \quad i \omega \rho_{f} \Phi_{1}^{(1)}-2 \mu\left(\Phi_{1, y y}^{(1)}-\Phi_{2, x y}^{(1)}\right)=\frac{K}{n}\left[\left(1-n+\alpha_{1} n\right) k_{1}^{2} \Phi_{1}^{(2)}\right. \\
& \left.\quad+\left(1-n+\alpha_{2} n\right) k_{2}^{2} \Phi_{2}^{(2)}\right]+2 i \omega \mu\left(\alpha_{1} \Phi_{1, y y}^{(2)}+\alpha_{2} \Phi_{2, y y}^{(2)}\right. \\
& \left.\quad-\alpha_{3} \Phi_{3, x y}^{(2)}-\alpha_{4} \Phi_{4, x y}^{(2)}\right) \\
& 2 \Phi_{1, x y}^{(1)}+\Phi_{2, y y}^{(1)}-\Phi_{2, x x}^{(1)}=-i \omega\left[2 \alpha_{1} \Phi_{1, x y}^{(2)}+2 \alpha_{2} \Phi_{2, x y}^{(2)}\right. \\
& \left.\quad+\alpha_{3}\left(\Phi_{3, y y}^{(2)}-\Phi_{3, x x}^{(2)}\right)+\alpha_{4}\left(\Phi_{4, y y}^{(2)}-\Phi_{4, x x}^{(2)}\right)\right] \\
& \quad i \omega \rho_{f} \Phi_{1}^{(1)}-2 \mu\left(\Phi_{1, y y}^{(1)}-\Phi_{2, x y}^{(1)}\right)=\frac{K}{n}\left[\left(1-n+\alpha_{1} n\right) k_{1}^{2} \Phi_{1}^{(2)}\right. \\
& \left.\quad+\left(1-n+\alpha_{2} n\right) k_{2}^{2} \Phi_{2}^{(2)}\right]-\frac{1}{1-n}\left[2 G \left(\Phi_{1, y y}^{(2)}+\Phi_{2 y y}^{(2)}\right.\right. \\
& \left.\left.\quad-\Phi_{3, x y}^{(2)}-\Phi_{4, x y}^{(2)}\right)-\lambda\left(k_{1}^{(2)} \Phi_{1}^{(2)}+k_{2}^{(2)} \Phi_{2}^{(2)}\right)\right] \\
& (1-n) \mu\left(2 \Phi_{1, x y}^{(1)}+\Phi_{2, y y}^{(1)}-\Phi_{2, x x}^{(1)}\right)=G\left(2 \Phi_{1, x y}^{(2)}+2 \Phi_{2, x y}^{(2)}+\Phi_{3, y y}^{(2)}\right. \\
& \left.\quad-\Phi_{3, x x}^{(2)}+\Phi_{4, y y}^{(2)}-\Phi_{4, x x}^{(2)}\right)
\end{aligned}
$$

Finally, at $y \rightarrow-\infty$, we get

$$
\Phi_{j}^{(2)} \rightarrow 0 ; \quad j=1,2,3,4
$$

because everything is quiescent at $y \rightarrow-\infty$.

\section{Solutions}

After omitting time factor $e^{-i \omega t}$, the linear water wave profile is taken as

$$
\eta(x)=\eta_{0} e^{i k_{0} x} ; \quad(0<x<\infty)
$$

where $\eta_{0}$ is the given amplitude and $k_{0}$ is the wave number to be determined by the dispersion relation. 
The solutions of velocity potentials $\Phi_{1}^{(1)}, \Phi_{2}^{(1)}$ for region (1) in Fig. 1 can be written as

$$
\begin{gathered}
\Phi_{1}^{(1)}(x, y)=\left[A_{0} \cosh k_{0}(h-y)+B_{0} \sinh k_{0}(h-y)\right] e^{i k_{0} x} \\
\Phi_{2}^{(1)}(x, y)=\left[\frac{a_{0}}{k_{0}^{2}} e^{K_{0} y}+\frac{b_{0}}{k_{0}^{2}} e^{-K_{0} y}\right] e^{i k_{0} x} \\
K_{0}^{2}=k_{0}^{2}-k_{w}^{2}, \quad \operatorname{Re}\left(K_{0}\right)<0
\end{gathered}
$$

with $A_{0}, B_{0}, a_{0}$, and $b_{0}$ to be determined by boundary conditions. The displacement potential, $\Phi_{j}, j=1,2,3,4$ for region (2) in Fig. 1 can be obtained as

$$
\begin{gathered}
\Phi_{j}^{(2)}=\frac{a_{j}}{k_{0}^{2}} e^{K_{j} y+i k_{0} x} ; \quad j=1,2,3,4 \\
K_{j}^{2}=k_{0}^{2}-k_{j}^{2}, \operatorname{Re}\left(K_{j}\right)>0 ; \quad j=1,2,3,4
\end{gathered}
$$

Again, the solutions of $a_{j}, j=1,2,3,4$ are to be found by boundary conditions.

After messy derivations, the solutions of $A_{0}, B_{0}, a_{j}, j=1$, $2,3,4, a_{0}, b_{0}$ are obtained and shown in Appendix I.

\section{Examinations of Fluid Viscosity and Rigid-Bed Permeability}

Note that, by introducing a parameter $\varepsilon_{0}$

$$
\varepsilon_{0}=\sqrt{\frac{2 \mu}{\rho_{f} \omega}} / h
$$

which means the ratio of Stokes boundary layer thickness near the channel bottom to water depth [for example, Dean and Dalrymple (1984)], and introducing a parameter $\varepsilon_{2}$

$$
\varepsilon_{2}=\frac{\mu \omega}{(G \text { or } K)}
$$

which means the comparison of viscous shear effect of fluid to deformation of the solid skeleton or to fluid compressibility in the porous medium, we find that, by referring to porous material handbooks, most porous material beds under a water wave may satisfy $\varepsilon_{0}=\sqrt{2 v / \rho_{f} \omega} / h<<1$ and $\varepsilon_{2}=\mu \omega /(G$ or $K)$ $<<1$. Under these conditions, the potential functions obtained from the laminar poroelastic media flow model are equivalent to those obtained from Biot's potential poroelastic media flow model. However, there are still some important physical discrepancies between a laminar and potential model due to fluid viscosity, even with the conditions of $\varepsilon_{0}<1$ and $\varepsilon_{2}<<1$.

By applying a typical combination of the water wave, fluid

TABLE 1. Water Wave Conditions, Fluid Properties, and Sand Bed Material

\begin{tabular}{l|c|c|c}
\hline \multicolumn{1}{c}{$\begin{array}{c}\text { Items } \\
(1)\end{array}$} & $\begin{array}{c}\text { Symbols } \\
(2)\end{array}$ & $\begin{array}{c}\text { Values } \\
(3)\end{array}$ & $\begin{array}{c}\text { Notations } \\
(4)\end{array}$ \\
\hline \multicolumn{4}{c}{$(a)$ Water wave } \\
\hline Depth & $h$ & 2.0 & $\mathrm{~m}$ \\
Wave height & $\eta_{0}$ & 0.2 & $\mathrm{~m}$ \\
Wave period & $T$ & 2.0 & $\mathrm{~s}$ \\
\hline \multicolumn{4}{c}{$(b)$ Water } \\
\hline Density & $\rho_{f}$ & 1,000 & $\mathrm{~kg} / \mathrm{m}^{3}$ \\
Bulk modulus & $K$ & $2.3 \times 10^{9}$ & $\mathrm{~N} / \mathrm{m}^{2}$ \\
Dynamic viscosity & $\mu$ & 0.001 & $\mathrm{Ns} / \mathrm{m}^{2}$ \\
\hline \multicolumn{4}{|c|}{$(c)$ Skeleton } \\
\hline Density & $\rho_{s}$ & 2,650 & $\mathrm{~kg} / \mathrm{m}^{3}$ \\
Lame's constant & $G$ & $5.0 \times 10^{8}$ & $\mathrm{~N} / \mathrm{m}^{2}$ \\
Lame's constant & $\lambda$ & $1.0 \times 10^{9}$ & $\mathrm{~N} / \mathrm{m}^{2}$ \\
Specific permeability & $k_{p}$ & $1.0 \times 10^{-12}$ & $\mathrm{~m}{ }^{2}$ \\
Porosity & $n$ & 0.4 & - \\
\hline \hline
\end{tabular}
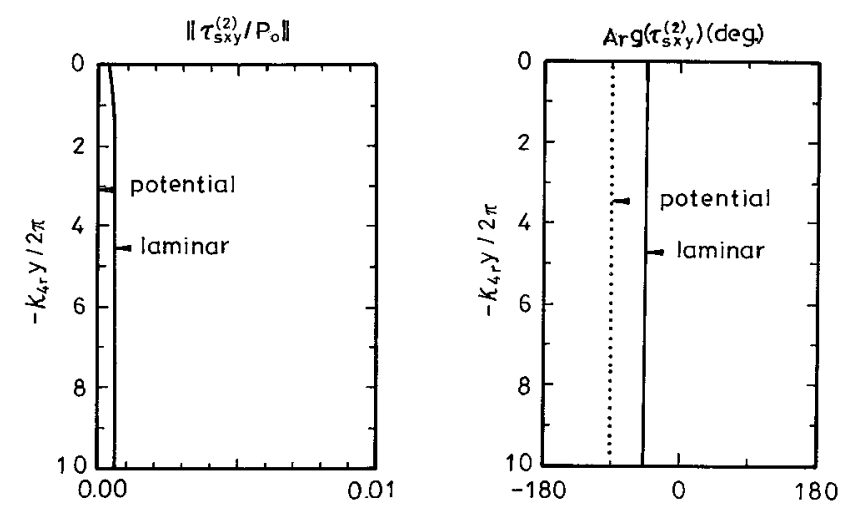

FIG. 2. Comparisons of Effective Stress $\tau_{s x y}^{(2)}$ of Laminar and Potential Models inside Sand Bed
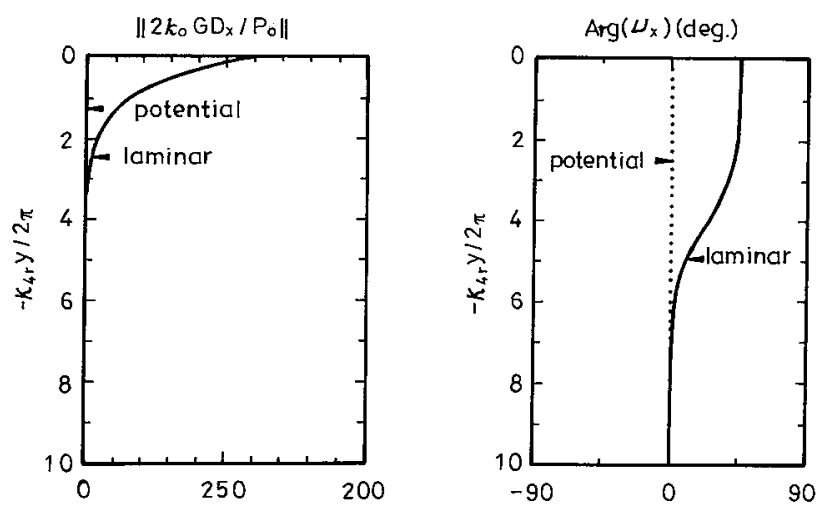

FIG. 3. Comparisons of Fluid Displacement $D_{x}$ of Laminar and Potential Models inside Sand Bed

property, and sand bed material indicated in Table $1\left[\varepsilon_{0}=\right.$ $\sqrt{2 \nu / \rho_{f} \omega} / h=4.0 \times 10^{-4}$ and $\varepsilon_{2}=\mu \omega /(G$ or $K)=6.3 \times 10^{-12}$ or $1.4 \times 10^{-12}$ ] to the solutions, $\tau_{s x y}^{(2)}, D_{x}$, and their phase angles inside the porous media are drawn in Figs. 2 and 3. The effective stress component of a solid skeleton, $\tau_{s x y}^{(2)}$, is nondimensionalized by perturbed pore pressure at the interface of the homogeneous channel fluid and porous bed (i.e., $P_{0}$ ), while the displacement component of the fluid, $D_{x}$, is nondimensionalized by $P_{0} / 2 k_{0} G$. The vertical axes of Figs. 2 and 3 are all normalized by $-2 \pi / k_{4 r}$, where $k_{4 r}$ is the real part of the wavenumber of the second transverse wave in the porous media. In Figs. 2 and 3, laminar solutions are the present result, while potential solutions are obtained from Huang and Song (1993), according to Biot's theory. (Note that $P^{(2)}, \tau_{s y y}^{(2)}$, $\tau_{s x x}^{(2)}, d_{x}, d_{y}$, and $D_{y}$ are almost identical for both the laminar and potential poroelastic models in this case.) From Fig. 2, we find that in the potential model $\tau_{s x y}^{(2)}=0$, while in the present laminar model $\tau_{s x y}^{(2)} \neq 0$, and the phase angle of laminar model is about $45^{\circ}$ ahead of potential model. On the other hand, Fig. 3 indicates that the unrealistic slipping at the channel bed interface in the potential model is removed and replaced by a more reasonable nonslip condition in the laminar model [recall the introduction concerning Beavers and Joseph's (1967) discussion of the boundary condition at the porous surface], and a boundary layer phenomena inside the porous bed is also revealed by this laminar poroelastic media flow model.

\section{Limiting Solution of Rigid Porous Bed}

The limiting solution when the porous bed is rigid is very useful for experimental comparison [this is the problem of Liu, Davis, and Downing (1996)]. Hence, this limiting case is worth discussing. By letting Lambe constants of elasticity of the solid skeleton be infinity (i.e., $G, \lambda \rightarrow \infty$ ), we immediately find that the first longitudinal displacement potential and the 
TABLE 2. Water Wave Conditions, Fluid Properties, and Porous Rock Material

\begin{tabular}{l|c|c|c}
\hline \multicolumn{1}{c|}{\begin{tabular}{c} 
Items \\
\hline \multicolumn{3}{c|}{$(1)$}
\end{tabular}} & $\begin{array}{c}\text { Symbols } \\
(2)\end{array}$ & $\begin{array}{c}\text { Values } \\
(3)\end{array}$ & $\begin{array}{c}\text { Notations } \\
(4)\end{array}$ \\
\hline Depth & $(a)$ Water wave & $\mathrm{m}$ \\
Wave height & $h$ & 2.0 & $\mathrm{~m}$ \\
Wave period & $\eta_{0}$ & 0.2 & $\mathrm{~s}$ \\
\hline \multicolumn{4}{c}{$(b)$ Water } \\
\hline Density & $\rho_{f}$ & 1,000 & $\mathrm{~kg} / \mathrm{m}^{3}$ \\
Bulk modulus & $K$ & $2.3 \times 10^{9}$ & $\mathrm{~N} / \mathrm{m}^{2}$ \\
Dynamic viscosity & $\mu$ & 0.001 & $\mathrm{Ns} / \mathrm{m}^{2}$ \\
\hline \multicolumn{4}{c}{$(c)$ Skeleton } \\
\hline Density & $\rho_{s}$ & 2,650 & $\mathrm{~kg} / \mathrm{m}^{3}$ \\
Lame's constant & $G$ & $5.0 \times 10^{10}$ & $\mathrm{~N} / \mathrm{m}^{2}$ \\
Lame's constant & $\lambda$ & $1.0 \times 10^{11}$ & $\mathrm{~N} / \mathrm{m}^{2}$ \\
Specific permeability & $k_{p}$ & $1.0 \times 10^{-12}$ & $\mathrm{~m}$ \\
Porosity & $n$ & 0.4 & - \\
\hline \hline
\end{tabular}
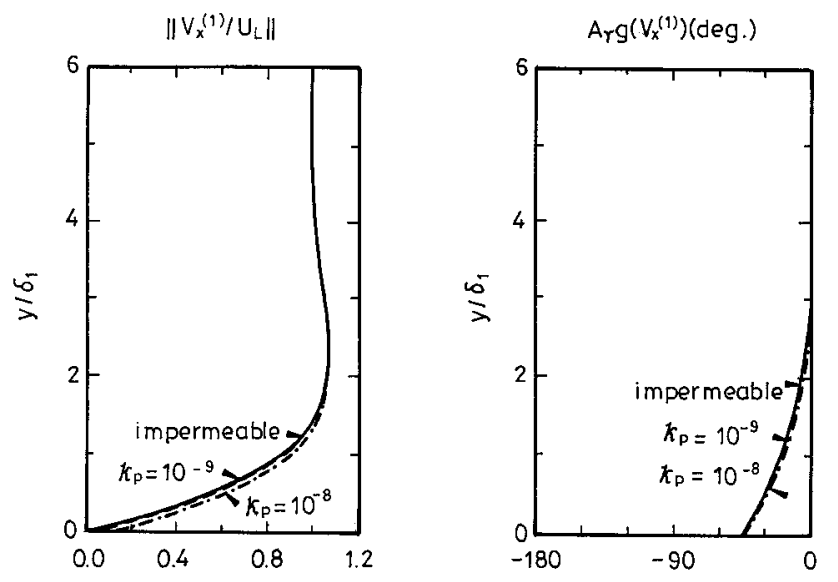

FIG. 4. Channel Flow Velocity $V_{x}^{(1)}$ of Water Wave near Rigid Porous Bed
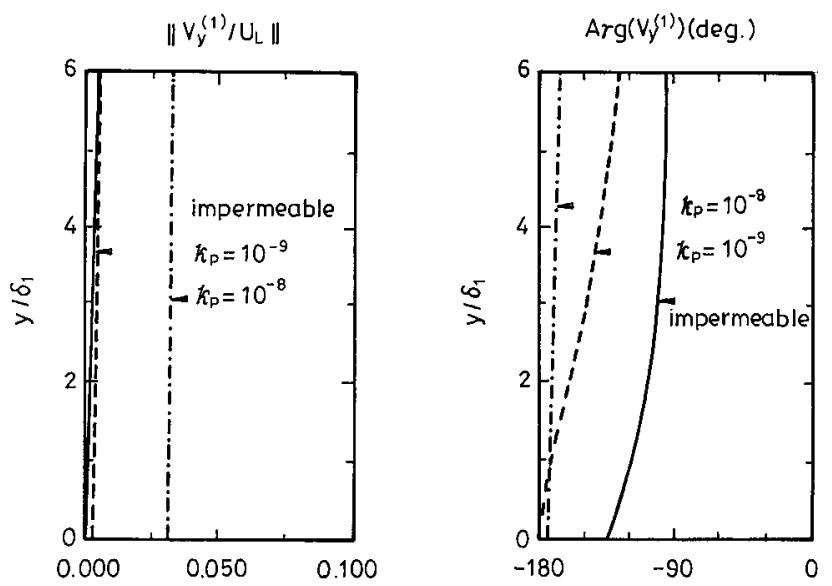

FIG. 5. Channel Flow Velocity $V_{y}^{(1)}$ of Water Wave near Rigid Porous Bed

first transverse displacement potential, $\Phi_{1}^{(2)}, \Phi_{3}^{(2)}$ vanish, and the solutions are listed in Appendix II.

Using the water wave, fluid property, and porous rock material given in Table 2 to simulate a water wave propagating over a rigid porous bed, we compare our solution in Appendix II to viscous water propagating over an impermeable rigid bed [e.g., Dean and Dalrymple (1984)]. Figs. 4 and 5 are the channel flow velocity components, $V_{x}^{(1)}, V_{y}^{(1)}$, normalized by $U_{L}$ and their phase angles versus nondimensional depth $y / \delta_{1} . U_{L}$ is the slipping velocity (i.e., velocity of the potential model at $y=$
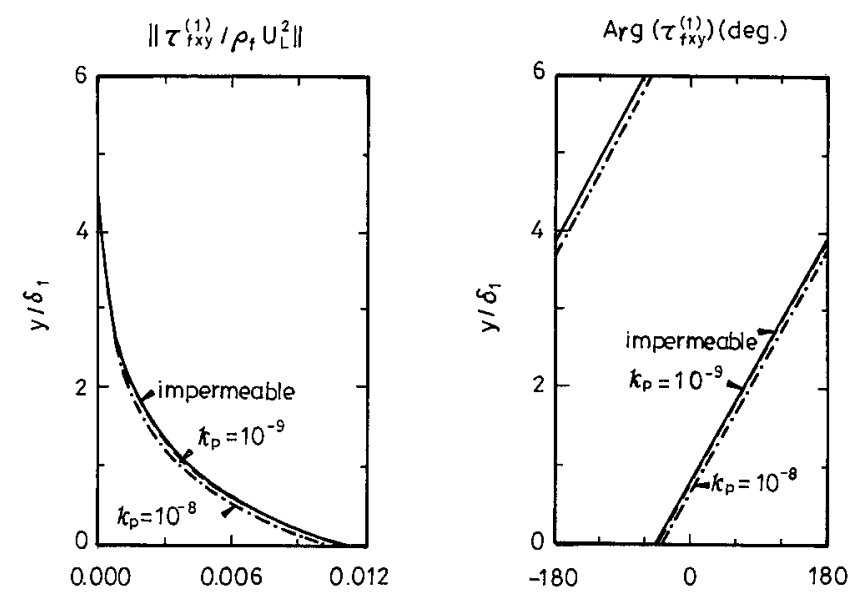

FIG. 6. Shear Stress $\tau_{f x y}^{(1)}$ of Water Wave near Rigid Porous Bed
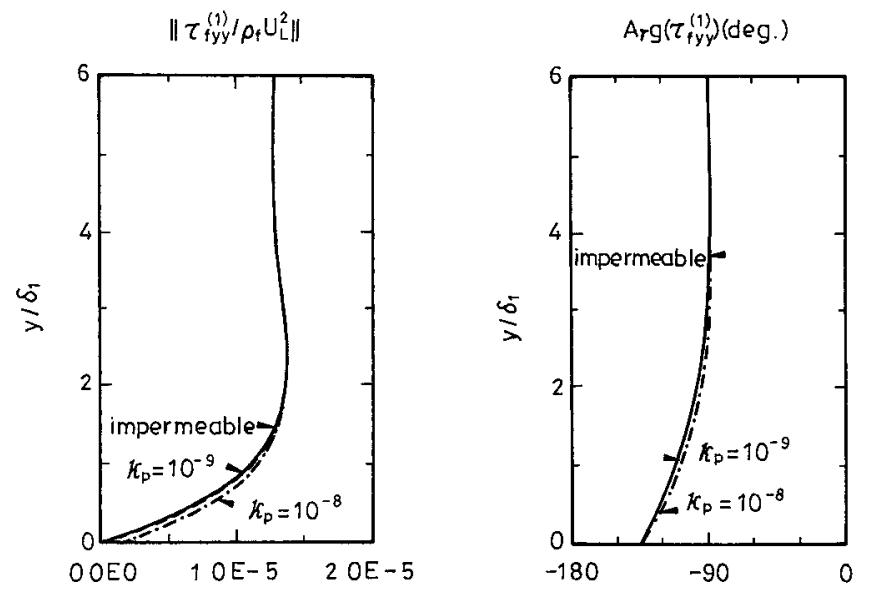

FIG. 7. Shear Stress $\tau_{f y y}^{(1)}$ of Water Wave near Rigid Porous Bed

$0)$, and $\delta_{1}$ is the Stokes boundary layer thickness defined as $\sqrt{2 v / \omega}$. Figs. 6 and 7 are the shear stress components of the channel fluid, $\tau_{f x y}^{(1)}$ and $\tau_{f y y}^{(1)}$, normalized by $\rho_{f} U_{L}^{2}$ and their phase angles versus $y / \delta_{1}$. In Figs. $4-7$, solid lines represent the conventional impermeable bed solution $\left(k_{p}=0\right)$, and dashed lines are the solutions of specific permeability $k_{p}=10^{-9} \mathrm{~m}^{2}$, while chain lines are the solutions of specific permeability $k_{p}=10^{-8}$ $\mathrm{m}^{2}$. Figs. 4, 6, and 7 show the variation of the Stokes boundary layer from impermeable bed to permeable bed. Fig. 4 is especially valuable for experimental verification of the velocity profile near the permeable bed, and Fig. 5 shows that, due to the permeable bed, there is a flux penetrating through the bed as long as the specific permeability is not zero.

\section{CONCLUSIONS}

In this study, we propose a laminar poroelastic flow model with sufficient boundary conditions. The present model is different from Biot's famous inviscid poroelastic model in further considering the shear stress of the flow itself inside the elastically defermable porous media. By simplification, the governing equations of the present model can recover the land subsidence equation, Brinkman equation, Darcy's law, and so forth. [Besides Biot (1962), further reviews and discussions on Biot's theory of poroelasticity can be found in the Maurice A. Biot Memorial Issue of the International Journal of Solids and Structures, volume 35 (1998).]

A viscous water wave passing over a poroelastic bed is solved analytically by applying this laminar poroelastic flow model through complicated algebraic work. It is found that, instead of two kinds of longitudinal waves and one kind of transverse wave, which can be obtained by Biot's theory of 
poroelasticity, an extra transverse wave due to fluid viscosity is discovered, according to the present new model. It is also found in the present study that not only can reasonable results that other methods can provide be maintained, but also more realistic nonslipping solutions near the porous bed can be given by our laminar poroelastic flow model than slipping solutions based on the conventional inviscid porous media flow model. The limiting case of a rigid bed solution, which is useful for experimental verification, is also provided in this study.

\section{APPENDIX I. SOLUTIONS OF WATER WAVE PASSING OVER POROELASTIC BED}

The following solutions of (71), (72), and (74) are obtained by substituting (71), (72), and (74) back to (61)-(68):

$$
\begin{gathered}
B_{0}=\frac{\delta_{0}}{\left(1+t_{3}\right) \sinh \xi}\left\{\left(1+t_{2}\right) \cosh \xi-\frac{1}{D}\left[\theta_{1} H_{2}\left(k_{1}^{2} / k_{0}^{2}\right)\right.\right. \\
\left.\left.-\theta_{2} H_{2}\left(k_{2}^{2} / k_{0}^{2}\right)+r_{1} H_{2}\right]\left(1-\frac{t_{1}}{\alpha_{0} \delta_{0}}\right)\right\} \\
a_{1}=\left(i \omega \rho_{f} n_{0} H_{2}\right)\left(-\alpha_{0} \delta_{0}+t_{1}\right) /(K D) \\
a_{4}=-\left(k_{0}^{2} / \omega\right)\left(E_{0}+E_{B} B_{0}\right)-E_{1} a_{1} \\
a_{0}=-(\omega / \Delta)\left(\Delta_{1} a_{1}+\Delta_{4} a_{4}\right)+\left(k_{0}^{2} / \Delta\right)\left(\delta_{0} \cosh \xi-\Delta_{0} B_{0}\right) \\
a_{2}=-\left(H_{1} / H_{2}\right) a_{1}-\left(H_{4} / H_{2}\right) a_{4} \\
a_{3}=-L_{1} a_{1}-L_{2} a_{2}-L_{4} a_{4} \\
b_{0}=\left(\delta_{a} / \delta_{b}\right) a_{0}-\delta_{a} k_{0}^{2} B_{0} /\left(K_{0} / k_{0}\right) \\
A_{0}=-\delta_{0}-\left(\delta_{a} a_{0}+\delta_{b} b_{0}\right) / k_{0}^{2}
\end{gathered}
$$

where

$$
\begin{aligned}
& \alpha_{0}=1-2\left(k_{0}^{2} / k_{w}^{2}\right) \\
& \delta_{0}=i g \eta_{0} /\left(\omega \alpha_{0}\right) \\
& \delta_{a}=2 i\left(K_{0} / k_{0}\right) e^{K_{0} h} /\left(\alpha_{0} k_{w}^{2} / k_{0}^{2}\right) \\
& \delta_{b}=-2 i\left(K_{0} / k_{0}\right) e^{-K_{0} h} /\left(\alpha_{0} k_{w}^{2} / k_{0}^{2}\right) \\
& /\left[1+\left(K_{3}^{2} / k_{0}^{2}\right)\right] \\
& /\left[1+\left(K_{3}^{2} / k_{0}^{2}\right)\right] \\
& -\left[\frac{\lambda}{2 G}\right]\left(\frac{k_{1}^{2}}{k_{0}^{2}}\right) \\
& -\left[\frac{\lambda}{2 G}\right]\left(\frac{k_{2}^{2}}{k_{0}^{2}}\right) \\
& H_{4}=i\left[1+\frac{(1-n) i \omega \mu \alpha_{4}}{G}\right]\left[\frac{1+K_{4}^{2} / k_{0}^{2}}{1+K_{3}^{2} / k_{0}^{2}}\left(\frac{K_{3}}{k_{0}}\right)-\left(\frac{K_{4}}{k_{0}}\right)\right] \\
& \Delta=-\left[2 \delta_{a} \cosh \xi+i\left(K_{0} / k_{0}\right)\left(1-\delta_{a} / \delta_{b}\right)\right] \\
& \Delta_{0}=\left[\sinh \xi+\delta_{a} \delta_{b} \cosh \xi /\left(K_{0} / k_{0}\right)-i \delta_{a}\right]
\end{aligned}
$$

$$
\begin{aligned}
& \Delta_{1}=\left[i \theta_{1} H_{2}-i \theta_{2} H_{1}+\theta_{3}\left(K_{3} / k_{0}\right)\left(L_{2} H_{1}-L_{1} H_{2}\right)\right] / H_{2} \\
& \Delta_{4}=\left[-i \theta_{2} H_{4}+\theta_{3}\left(K_{3} / k_{0}\right)\left(L_{2} H_{4}-L_{4} H_{2}\right)+\theta_{4}\left(K_{4} / k_{0}\right) H_{2}\right] / H_{2} \\
& q_{0}=4 \delta_{\alpha} \sinh \xi-i\left[1+\left(K_{0}^{2} / k_{0}^{2}\right)\right]\left(1+\delta_{a} / \delta_{b}\right) \\
& E=-2 i \alpha_{2}\left(K_{2} / k_{0}\right)\left(H_{4} / H_{2}\right)+\alpha_{3}\left[1+\left(K_{3}^{2} / k_{0}^{2}\right)\right]\left(L_{2} H_{4} / H_{2}-L_{4}\right) \\
& +\alpha_{4}\left[1+\left(K_{4}^{2} / k_{0}^{2}\right)\right]-q_{0} \Delta_{4} / \Delta \\
& E_{0}=\left(2 \delta_{0} \sinh \xi+\delta_{0} \cosh \xi q_{0} / \Delta\right) / E \\
& E_{1}=\left\{2 i \alpha_{1}\left(K_{1} / k_{0}\right)-2 i \alpha_{2}\left(K_{2} / k_{0}\right)\left(H_{1} / H_{2}\right)\right. \\
& \left.+\alpha_{3}\left[1+\left(K_{3}^{2} / k_{0}^{2}\right)\right]\left(L_{2} H_{1} / H_{2}-L_{1}\right)-q_{0} \Delta_{1} / \Delta\right\} / E \\
& E_{B}=-\left\{2 \cosh \xi-i\left[1+\left(K_{0}^{2} / k_{0}^{2}\right)\right] \delta_{a} /\left(K_{0} / k_{0}\right)\right. \\
& \left.+2 \delta_{a} \delta_{b} \sinh \xi /\left(K_{0} / k_{0}\right)+q_{0} \Delta_{0} / \Delta\right\} / E \\
& q_{1}=i \omega \alpha_{1}\left(2 \mu K_{1}^{2} / k_{0}^{2}\right)(n / K) \\
& q_{2}=i \omega \alpha_{2}\left(2 \mu K_{2}^{2} / k_{0}^{2}\right)(n / K) \\
& q_{3}=2 \omega \mu \alpha_{3}\left(K_{3} / k_{0}\right)(n / K) \\
& q_{4}=2 \omega \mu \alpha_{4}\left(K_{4} / k_{0}\right)(n / K) \\
& D_{a}=-\alpha_{0} \delta_{a} \cosh \xi+2 i\left(K_{0} / k_{0}\right)\left(k_{0}^{2} / k_{w}^{2}\right) \\
& D_{b}=-\alpha_{0} \delta_{b} \cosh \xi-2 i\left(K_{0} / k_{0}\right)\left(k_{0}^{2} / k_{w}^{2}\right) \\
& r_{0}=-\left(\theta_{2} k_{2}^{2} / k_{0}^{2}+q_{2}\right)\left(H_{4} / H_{2}\right)+q_{3}\left(L_{2} H_{4} / H_{2}-l_{4}\right)+q_{4} \\
& r_{1}=q_{1}-q_{2} H_{1} / H_{2}+q_{3}\left(l_{2} H_{1} / H_{2}-L_{1}\right)-r_{0} E_{1} \\
& +\left(i \omega \rho_{f} n / k_{0}^{2} K\right)\left(D_{a}+D_{b} \delta_{a} / \delta_{b}\right)\left(\Delta_{1} / \Delta-E_{1} \Delta_{4} / \Delta\right) \\
& \left.r_{2}=\left(D_{a}+D_{b} \delta_{a} / \delta_{b}\right)\right)\left(\delta_{0} \cosh \xi+E_{0} \Delta_{4}\right) / \Delta-i r_{0} E_{0} k_{0}^{2} K /\left(\omega^{2} \rho_{f} n\right)
\end{aligned}
$$

$$
r_{3}=-D_{b} \delta_{a} /\left(K_{0} / k_{0}\right)-\left(D_{a}+D_{b} \delta_{a} / \delta_{b}\right)\left(\Delta_{0}-E_{B} \Delta_{4}\right) / \Delta
$$$$
-i r_{0} E_{B} k_{0}^{2} K /\left(\omega^{2} \rho_{f} n\right)
$$

$$
S_{0}=\theta_{2}\left(K_{2} / k_{0}\right)\left(H_{4} / H_{2}\right)+i \theta_{3}\left(L_{2} H_{4} / H_{2}-L_{4}\right)+i \theta_{4}
$$

$S_{1}=S_{0} E_{1}-i\left[i\left(1+\delta_{a} / \delta_{b}\right)-2 \delta_{a} \sinh \xi\right]\left(\Delta_{1}-E_{1} \Delta_{4}\right) / \Delta$

$S_{2}=i S_{0} E_{0}-\left[i\left(1+\delta_{a} / \delta_{b}\right)-2 \delta_{a} \sinh \xi\right]\left(\delta_{0} \cosh \xi+E_{0} \Delta_{4}\right) / \Delta$

$$
\begin{aligned}
S_{3} & =i S_{0} E_{B}-\left[i\left(1+\delta_{a} / \delta_{b}\right)-2 \delta_{a} \sinh \xi\right]\left(\Delta_{0}-E_{B} \Delta_{4}\right) / \Delta \\
& -\left(\delta_{a} \delta_{b} \sinh \xi-i \delta_{a}\right) /\left(K_{0} / k_{0}\right) \\
D & =\left[\theta_{1}\left(k_{1}^{2} / k_{0}^{2}\right) H_{2}-\theta_{2}\left(k_{2}^{2} / k_{0}^{2}\right) H_{1}+r_{1} H_{2}\right]\left(\cosh \xi-S_{3}\right) \\
& +\left(\omega^{2} \rho_{f} n / k_{0}^{2} K\right)\left[\theta_{1}\left(K_{1} / k_{0}\right) H_{2}-\theta_{2}\left(K_{2} / k_{0}\right) H_{1}\right.
\end{aligned}
$$$$
\left.-i \theta_{3}\left(L_{2} H_{1}-L_{1} H_{2}\right)+S_{1} H_{2}\right]\left(\alpha_{0} \sinh \xi+r_{3}\right)
$$$$
t_{1}=\left(S_{3} \alpha_{0} \delta_{0}+r_{2}\right) \cosh \xi+\left(r_{3} \delta_{0}+S_{2} \alpha_{0}\right) \sinh \xi+\left(r_{3} S_{2}-r_{2} S_{3}\right)
$$

$$
\theta_{i}=1-n+\alpha_{i} n ; \quad i=1, \ldots, 4
$$

Finally, by using the remaining boundary condition (60), the dispersion relation is obtained as

$$
\begin{aligned}
1 & +\frac{1-K_{0}^{2} / k_{0}^{2}}{1+K_{0}^{2} / k_{0}^{2}}\left(\frac{g k_{0}}{\alpha_{0} \omega^{2} \sinh \xi}\right)\left\{\left(1+t_{2}\right) \cosh \xi-\frac{1}{D}\left[\theta_{1} H_{2}\left(k_{1}^{2} / k_{0}^{2}\right)\right.\right. \\
& \left.\left.-\theta_{2} H_{2}\left(k_{2}^{2} / k_{0}^{2}\right)+r_{1} H_{2}\right]\left(1-\frac{t_{1}}{\alpha_{0} \delta_{0}}\right)\right\} /\left(1+t_{3}\right)=0
\end{aligned}
$$


Eq. (124) is used to determine wavenumber $k_{0}$.

\section{APPENDIX II. SOLUTIONS OF WATER WAVE PASSING OVER RIGID POROUS BED}

By letting $G, \lambda \rightarrow \infty$, the limiting solutions are obtained as $\Phi_{1}^{(1)}(x, y)=\left[A_{0} \cosh k_{0}(h-y)+B_{0} \sinh k_{0}(h-y)\right] e^{i k_{0} x}$

$$
\begin{gathered}
\left.\Phi_{2}^{(1)}(x, y)=\left[a_{0} / k_{0}^{2}\right) e^{K_{0} y}+\left(b_{0} / k_{0}^{2}\right) e^{-K_{0} y}\right] e^{i k_{0} x} \\
\Phi_{2}^{(2)}(x, y)=\left[a_{2} / k_{0}^{2}\right) e^{K_{2} y} e^{i k_{0} x} \\
\Phi_{4}^{(2)}(x, y)=\left[a_{4} / k_{0}^{2}\right) e^{K_{4} y} e^{i k_{0} x}
\end{gathered}
$$

with

$$
\begin{gathered}
B_{0}=\frac{\delta_{0}}{\left(1+t_{3}\right) \sinh \xi}\left\{\left(1+t_{2}\right) \cosh \xi-\frac{1}{D}\left[\left(k_{2}^{2} / k_{0}^{2}\right)+r_{1}\right]\right. \\
\left.\cdot\left(1-\frac{t_{1}}{\alpha_{0} \delta_{0}}\right)\right\} \\
a_{2}=\omega^{2} \rho_{f}\left(-\alpha_{0} \delta_{0}+t_{1}\right) /(K D) \\
a_{4}=-E_{2} a_{2}-E_{B} k_{0}^{2} B_{0}-E_{0} k_{0}^{2} \\
a_{0}=\left(\delta_{0} \cosh \xi k_{0}^{2}+n_{0} a_{2}-\Delta_{4} a_{4}-\Delta_{0} k_{0}^{2} B_{0}\right) / \Delta \\
b_{0}=\left(\delta_{a} / \delta_{b}\right) a_{0}-\delta_{a} k_{0}^{2} B_{0} /\left(K_{0} / k_{0}\right) \\
A_{0}=-\delta_{0}-\left(\delta_{a} a_{0}+\delta_{b} b_{0}\right) / k_{0}^{2}
\end{gathered}
$$

where

$$
\begin{aligned}
& k_{2}^{2}=\frac{i \omega \rho_{f}-F(\kappa) \mu n}{(i K / \omega)+2 \mu} \\
& k_{4}^{2}=\frac{i \omega \rho_{f}-F(\kappa) \mu n}{\mu} \\
& K_{2}^{2}=k_{0}^{2}-k_{2}^{2}, \quad \operatorname{Re}\left(K_{2}\right)>0 \\
& K_{4}^{2}=k_{0}^{2}-k_{4}^{2}, \quad \operatorname{Re}\left(K_{4}\right)>0 \\
& \alpha_{0}=1-2\left(k_{0}^{2} / k_{w}^{2}\right) \\
& \delta_{0}=i g \eta_{0} /\left(\omega \alpha_{0}\right) \\
& \delta_{a}=2 i\left(K_{0} / k_{0}\right) e^{K_{0} h} /\left(\alpha_{0} k_{w}^{2} / k_{0}^{2}\right) \\
& \delta_{b}=-2 i\left(K_{0} / k_{0}\right) e^{-K_{0} h} /\left(\alpha_{0} k_{w}^{2} / k_{0}^{2}\right) \\
& \Delta=-\left[2 \delta_{a} \cosh \xi+i\left(K_{0} / k_{0}\right)\left(1-\delta_{a} \delta_{b}\right)\right] \\
& \Delta_{0}=\left[\sinh \xi+\delta_{a} \delta_{b} \cosh \xi /\left(K_{0} / k_{0}\right)-i \delta_{a}\right] \\
& \Delta_{4}=i n K_{2} / k_{0} \\
& q_{0}=4 \delta_{a} \sinh \xi-i\left[1+\left(K_{0}^{2} / k_{0}^{2}\right)\right]\left(1+\delta_{a} / \delta_{b}\right) \\
& E=i\left[1+\left(K_{2}^{2} / k_{0}^{2}\right)\right]-q_{0} \Delta_{4} / \Delta \\
& E_{0}=\left(2 \delta_{0} \sinh \xi+\delta_{0} \cosh \xi q_{0} / \Delta\right) E \\
& E_{2}=\left[\left(n q_{0} / \Delta\right)-2\left(K_{1} / k_{0}\right)\right] / E \\
& q_{2}=\left[2 i \omega \mu\left(K_{2}^{2} / k_{0}^{2}\right)-i \omega \mu^{\prime}\left(k_{2}^{2} / k_{0}^{2}\right)\right] / K \\
& q_{4}=2 \omega \mu\left(K_{4} / k_{0}\right) / K \\
& D_{a}=-\alpha_{0} \delta_{a} \cosh \xi+2 i\left(K_{0} / k_{0}\right)\left(k_{0}^{2} / k_{w}^{2}\right) \\
& D_{b}=-\alpha_{0} \delta_{b} \cosh \xi-2 i\left(K_{0} / k_{0}\right)\left(k_{0}^{2} / k_{w}^{2}\right)
\end{aligned}
$$

$$
r_{1}=q_{2}-E_{2} q_{4}-\left(\omega^{2} \rho_{f} / k_{0}^{2} K\right)\left(D_{a}+D_{b} \delta_{a} / \delta_{b}\right)\left(n / \Delta+E_{2} \Delta_{4} / \Delta\right)
$$

$$
r_{2}=\left(D_{a}+D_{b} \delta_{a} / \delta_{b}\right)\left(\delta_{0} \cosh \xi+E_{0} \Delta_{4}\right) / \Delta+q_{4} E_{0} k_{0}^{2} K /\left(\omega^{2} \rho_{f}\right)
$$

$$
\begin{array}{r}
r_{3}=-D_{b} \delta_{a} /\left(K_{0} / k_{0}\right)-\left(D_{a}+D_{b} \delta_{a} / \delta_{b}\right)\left(\Delta_{0}-E_{B} \Delta_{4}\right) / \Delta \\
+q_{4} E_{B} k_{0}^{2} K /\left(\omega^{2} \rho_{f}\right) \\
S_{0}=2 \delta_{a} \sinh \xi-i\left(1+\delta_{a} / \delta_{b}\right) \\
S_{1}=i n_{0} E_{2}-S_{0}\left(n+\Delta_{4} E_{2}\right) / \Delta \\
S_{2}=S_{0}\left(\delta_{0} \cosh \xi+\Delta_{4} E_{0}\right) / \Delta-i n E_{0}
\end{array}
$$

$S_{3}=\left(\delta_{a} \delta_{b} \sinh \xi-i \delta_{a}\right) /\left(K_{0} / k_{0}\right)+S_{0}\left(\Delta_{0}-\Delta_{4} E_{B}\right) / \Delta+i n E_{B}$

$$
\begin{aligned}
D & =\left[\left(k_{1}^{2} / k_{0}^{2}\right)+r_{1}\right]\left(\cosh \xi+S_{3}\right) \\
& +\left(S_{1}+n K_{2} / k_{0}\right)\left(\alpha_{0} \sinh \xi+r_{3}\right)\left(\omega^{2} \rho_{f} / k_{0}^{2} K\right)
\end{aligned}
$$$$
t_{1}=\left(r_{2}-S_{3} \alpha_{0} \delta_{0}\right) \cosh \xi+\left(r_{3} \delta_{0}+S_{2} \alpha_{0}\right) \sinh \xi+\left(r_{3} S_{2}+r_{2} S_{3}\right)
$$

$$
\begin{gathered}
t_{2}=-r_{2} /\left(\delta_{0} \alpha_{0} \cosh \xi\right) \\
t_{3}=r_{3} /\left(\alpha_{0} \sinh \xi\right)
\end{gathered}
$$$$
\xi=k_{0} h
$$

and the dispersion relation is

$$
\begin{gathered}
1+\frac{1-K_{0}^{2} / k_{0}^{2}}{1+K_{0}^{2} / k_{0}^{2}}\left(\frac{g k_{0}}{\alpha_{0} \omega^{2} \sinh \xi}\right)\left\{\left(1+t_{2}\right) \cosh \xi-\frac{1}{D}\left[\left(k_{2}^{2} / k_{0}^{2}\right)+r_{1}\right]\right. \\
\left.\cdot\left(1-\frac{t_{1}}{\alpha_{0} \delta_{0}}\right)\right\} /\left(1+t_{3}\right)=0
\end{gathered}
$$

\section{ACKNOWLEDGMENT}

The preliminary part of this study is sponsored by the National Science Council of the Republic of China, under grant NSC80-0410-E002-66.

\section{APPENDIX III. REFERENCES}

Beavers, G. S., and Joseph, D. D. (1967). "Boundary conditions at a naturally permeable wall." J. Fluid Mech., 30, 197-207.

Biot, M. A. (1962). "Mechanics of deformation and acoustic propagation in porous media." J. Appl. Physics, 33, 1482-1498.

Biot, M. A. (1956). "Theory of propagation of elastic waves in a fluidsaturated porous solid. II: Higher frequency range." JASA, 28, 179191.

Brinkman, H. C. (1947). "A calculation of the viscous force exerted by a flowing fluid on a dense swarm of particles." Appl. Scientific Res., The Hague, The Netherlands, A1, 27-34.

Chen, T. W., Huang, L. H., and Song, C. H. (1997). "Dynamic response of poroelastic bed to nonlinear water waves." J. Engrg. Mech., ASCE, 123(10), 1041-1049.

Dean, R. G., and Dalrymple, R. A. (1984). Water wave mechanics for engineers and scientists. Prentice-Hall, Englewood Cliffs, N.J.

Deresiewicz, R., and Skalak, R. (1962). "On uniqueness in dynamic poroelasticity." Bull. Seismological Soc. of Am., 53, 783-788.

Huang, L. H., Chiang, I. L., and Song, C. H. (1996). "Laminar channel flow passing over porous bed." Hydrodynamics, A. T. Chwang, J. H. W. Lee, and D. Y. C. Leung, eds., Balkema, Rotterdam, The Netherlands, 1341-1346.

Huang, L. H., and Chwang, A. T. (1990). “Trapping and absorption of sound waves. II: A sphere covered with a porous layer." Wave Motion, 12, 401-414.

Huang, L. H., and Song, C. H. (1993). "Dynamic response of poroelastic bed to water waves." J. Hydr. Engrg., ASCE, 119(9), 1003-1020.

Hunt, J. N. (1959). "On the damping of gravity waves propagated over a permeable surface.” J. Geophys. Res., 64, 437-442.

Katsube, N., and Carroll, M. M. (1987). "The modified mixture theory for fluid-filled porous materials: Theory." J. Appl. Mech., 54, 35-40.

Liu, L. F. (1973). "Damping of water waves over porous bed." J. Hydr. Div., ASCE, 99, 2263-2271. 
Liu, L. F., Davis, M. H., and Downing, S. (1996). "Wave-induced boundary layer flows above and in a permeable bed." J. Fluid Mech., Cambridge, England, 325, 195-218.

Mei, C. C., and Foda, M. A. (1981). "Wave-induced responses in a fluid filled poroelastic solid with a free surface-A boundary layer theory." Geophys. J. Royal Astronomical Soc., 66, 597-631.

Murray, J. D. (1965). "Viscous damping of gravity waves over a permeable bed." J. Geophys. Res., 70, 2325-2331.

Verruijt, A. (1969). "Elastic storage of aquifers." Flow through porous media, R. J. M. de Weist, ed., Academic, New York.

\section{APPENDIX IV. NOTATION}

The following symbols are used in this paper:

$\mathbf{D}=$ displacement vector of fluid in porous bed;

$\mathbf{d}=$ displacement vector of solid skeleton;

$E=$ internal energy of system;

$G=$ Lambe constant of elasticity;

$g=$ gravitational acceleration;

$K=$ static bulk modulus of compressibility of fluid;

$k_{0}=$ wave number of incoming water wave;

$k_{1}, k_{2}, k_{3}, k_{4}=$ wave numbers in porous bed;

$k_{p}=$ specific permeability of porous medium;

$n=$ porosity;

$P=$ total pressure of fluid;

$P_{0}=$ perturbed pore pressure at channel bed interface;

$P^{(2)}=$ perturbed pore pressure inside porous bed;

$P^{\prime}=$ perturbed fluid pressure caused by fluid dilatation;

$Q=$ heat added to system;

$\mathbf{U}=$ velocity vector of fluid;

$U_{L}=$ slipping velocity;
$W=$ work added to system;

$\delta_{1}=$ Stokes boundary layer thickness;

$\varepsilon_{0}=$ parameter of Stokes boundary layer thickness to water depth;

$\varepsilon_{2}=$ parameter of viscous shear effect to deformation of solid or to fluid compressibility;

$\eta=$ free surface displacement;

$\eta_{0}=$ amplitude of given water wave;

$\lambda=$ Lambe constant of elasticity;

$\rho_{f}=$ density of fluid;

$\rho_{s}=$ density of solid;

$\boldsymbol{\sigma}_{f}=$ stress tensor of fluid;

$\boldsymbol{\sigma}_{f}^{0}=$ stress tensor of steady streaming flow;

$\boldsymbol{\sigma}_{f}^{\prime}=$ stress tensor of unsteady viscous flow;

$\boldsymbol{\sigma}_{s}=$ stress tensor of solid skeleton;

$\boldsymbol{\sigma}_{s}^{0}=$ stress tensor of stationary deformed solid skeleton;

$\boldsymbol{\sigma}_{s}^{\prime}=$ stress tensor of unsteady disturbed solid skeleton;

$\boldsymbol{\tau}_{f}^{(2)}=$ shear stress tensor of fluid in porous bed;

$\boldsymbol{\tau}_{f}^{\prime}=$ shear stress tensor of viscous flow;

$\boldsymbol{\tau}_{s}^{(2)}=$ effect stress tensor of solid skeleton;

$\boldsymbol{\tau}_{s}^{\prime}=$ effect stress tensor of unsteadily disturbed solid skeleton;

$\Phi_{1}=$ irrotational velocity potential of channel flow;

$\Phi_{2}=$ rotational part of velocity potential of channel flow;

$\Phi_{1}^{(2)}, \Phi_{2}^{(2)}=$ irrotational displacement potential of porous bed; $\Phi_{3}^{(2)}, \Phi_{4}^{(2)}=$ rotational parts of displacement potential of porous bed; and

$\omega=$ angular frequency. 